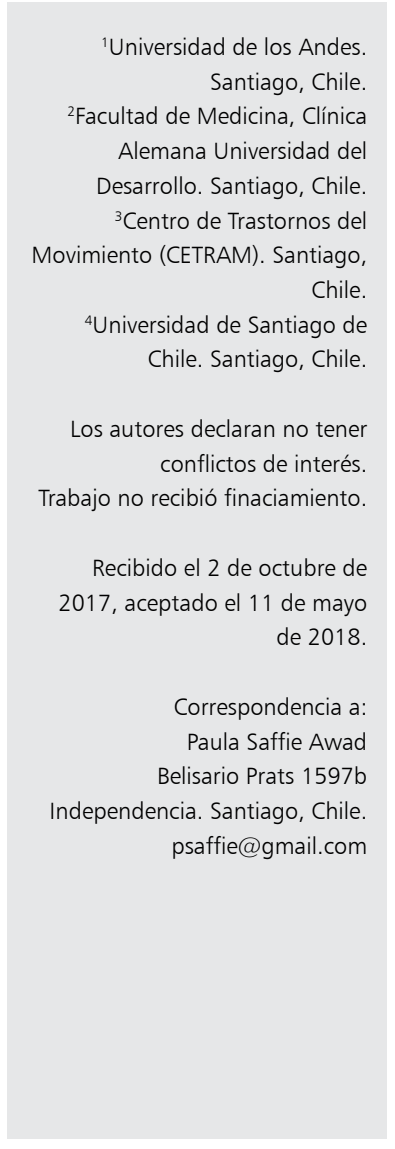

\section{Características clínicas de 63 pacientes con ataxia}

\author{
PAULA SAFFIE AWAD ${ }^{1,3}$, FELIPE VIAL UNDURRAGA ${ }^{2,3}$, \\ PEDRO CHANÁ-CUEVAS ${ }^{3,4}$
}

\section{Clinical features of 63 patients with ataxia}

Background: Ataxia can be classified as genetic, sporadic or acquired. Aim: To report the clinical features of a group of patients with ataxia. Material and Methods: Review of medical records of patients consulting in a specialized center in movement disorders. Those records in which the diagnosis of "ataxia" or "ataxic syndrome" appeared, were selected for the review. Results: Of 4,282 records surveyed, the diagnosis of ataxia appeared in 95. After eliminating repeated or incomplete records, 63 were reviewed. Results: Ataxia was sporadic, genetic and acquired in 27, 22 and 14 patients, respectively. The mean age at presentation for genetic, acquired and sporadic ataxia was 24, 46 and 53 years respectively. All autosomal dominant ataxias were type 3 spinocerebellar ataxia (SCA). Friedrich's ataxia was the most common recessive form. Most sporadic forms of ataxia were multiple system atrophy with predominant cerebellar ataxia (MSA-C) subtype. Conclusions: Considering the heterogeneity of patients with ataxia, we propose a method to approach them.

(Rev Med Chile 2018; 146: 702-707)

Key words: Cerebellar Ataxia; Friedreich Ataxia; Machado-Joseph Disease; Spinocerebellar Ataxias.

\section{L} a palabra ataxia viene del griego y significa literalmente sin orden (taxos = orden). En medicina se usa para denominar un síndrome que se caracteriza por las siguientes alteraciones motoras; incoordinación, dismetría, disdiadococinesia, y aumento de base de sustentación en la marcha. También se usa para designar un grupo específico de enfermedades neurodegenerativas cuyo síntoma principal es la ataxia ${ }^{1}$.

Las ataxias pueden clasificarse según su etiología en tres grupos; neurodegenerativas hereditarias (genéticas), neurodegenerativas no hereditarias (esporádicas), no neurodegenerativas (adquiridas) ${ }^{1}$.

El grupo de las ataxias neurodegenerativa tanto genéticas como esporádicas, son un motivo poco frecuente de consulta en una clínica de trastornos del movimiento $(1,9 \%)^{2}$. Este grupo de enfermedades, constituyen un desafío diagnóstico importante por varias razones; la sobreposición de síntomas y signos que habitualmente no se limitan a compromiso cerebeloso, la falta de especificidad de los exámenes generales, y en nuestro país se agrega la falta de acceso al estudio genético.

En Chile, no hay estudios epidemiológicos sobre ataxias neurodegenerativas y es difícil encontrar estudios del tema en general. En estudios internacionales dentro de las causas neurodegenerativas no hereditarias la causa más frecuente es la atrofia multisistémica tipo cerebeloso (AMS-C) ${ }^{3}$, en el caso de las ataxias hereditarias, la más común es la ataxia de Friedreich ${ }^{4}$. Estudios de prevalencia para ataxias hereditarias la describen entre $0,31 \mathrm{y}$ 41 por 100.000 habitantes $^{5}$.

El objetivo de este trabajo fue hacer un estudio retrospectivo sobre los pacientes que se presentan con ataxia en un centro especializado en trastornos del movimiento, con un especial énfasis en la caracterización de los casos de ataxia de etiología neurodegenerativa. 


\section{Pacientes y Método}

Este estudio se realizó en el Centro de Trastornos del Movimiento (CETRAM). El CETRAM es una organización no gubernamental sin fines de lucro dedicada a la atención de personas con trastornos del movimiento, investigación y docencia. Los pacientes son evaluados en este centro por un equipo de neurólogos y otros profesionales de la salud especializados en esta área de la neurología.

En este estudio retrospectivo se revisaron un total de 4.282 fichas clínicas (electrónicas y en papel) de los pacientes que consultaron en el centro entre el 1 de enero de 2004 hasta el 1 de enero de 2016.

Se seleccionan aquellas fichas en las que aparece la palabra "ataxia" o "síndrome atáxico" tanto en antecedentes, diagnóstico y evolución. Se obtienen 95 fichas, 24 de papel y 71 electrónicas. Se seleccionaron luego los casos en los cuales la ataxia era el síntoma principal. Se eliminaron fichas duplicadas o incompletas quedando finalmente con 63 casos.

Se registran variables demográficas; edad al inicio del cuadro clínico, sexo, antecedentes familiares, hallazgos del examen físico neurológico, compromiso autonómico, cognitivo y exámenes solicitados. La información obtenida se almacenó en una base de datos estandarizada y confidencial.

La clasificación se realizó de acuerdo al mejor criterio del médico tratante, historia y exámenes disponibles. De tal modo, fueron clasificados como de origen genético los casos en los cuales se encontró una mutación y/o casos con familiares afectados, como esporádicos casos de ataxia progresiva sin criterios como para clasificarlos de genéticos, y adquiridos los casos no progresivos y/o con evidencia de patología no neurodegenerativa.

Los resultados se expresaron en porcentajes o medias \pm desviaciones estándar según correspondía. Para el análisis multifactorial, se realizó análisis de varianza (ANOVA) y luego corrección postHoc con método de Tukey. Se utilizó el paquete estadístico SPSS versión 22 para Windows. Se consideró estadísticamente significativo un $\mathrm{p}<0,05$.

Este estudio cuenta con la aprobación del comité de ética de la institución.

\section{Resultados}

Se encontraron 63 casos con ataxia, el 52\% eran hombres, con un promedio de edad de inicio de los síntomas a las 41,3 años. De acuerdo a la clasificación, se encontraron 27 casos de ataxias esporádica, 22 casos de ataxia genética y 14 de ataxia adquirida. La media de edad de presentación para los distintos grupos de ataxia fue 23,7 años para las ataxias de origen genético, 45,7 para las adquiridas y 52,9 para los esporádica. El análisis con ANOVA para edad versus clasificación resultó estadísticamente significativo $(\mathrm{F}=17,5 ; \mathrm{p}<0,001)$. $\mathrm{El}$ análisis postHoc mostró diferencia entre causa genética versus adquirida $(\mathrm{p}=0,001)$ y genética versus esporádica $(\mathrm{p}<0,001)$. No se encontró diferencias por sexo (Tabla 1 ).

Del total de pacientes incluidos en el estudio hubo $20(31,7 \%)$ en los cuales no se llegó a diagnóstico etiológico.

Las características de los casos de ataxia adquirida se resumen en la Tabla 2, destacando como los diagnósticos más frecuentes, las ataxias psicogénicas y secundarias a polineuropatía.

Tabla 1. Características demográficas y clínicas

\begin{tabular}{|lllll|}
\hline Resumen de casos de ataxia & $\mathbf{n}$ & $\mathbf{( \% )}$ & & \\
\hline Casos & 63 & & & \\
\hline Hombres & 33 & $(52 \%)$ & & \\
\hline Edad de inicio & 41,3 & $(3-81)$ & Media edad de inicio & Hombres (\%) \\
\hline Edad de diagnóstico & 50 & $(16-64)$ & $45,7(18-81)$ & 42,8 \\
\hline Clasificación & & & $52,9(23-80)$ & 55,5 \\
\hline Adquirida & 14 & $(22,2)$ & $23,7(3-59)$ & 54,5 \\
\hline Esporádica & 27 & $(42,8)$ & & \\
\hline Genética & 22 & $(34,9)$ & & \\
\hline
\end{tabular}


En relación a las ataxias neurodegenerativas, los diagnósticos desagregados por sexo, se presentan en la Figura 1. No se ven grandes diferencias por sexo. En las ataxias genéticas destacan como causas más frecuentes SCA3 (7 casos) y ataxia de Friedreich (7 casos). Se reportan también 2 casos de ataxia autosómica recesiva de Charlevoix Saguenay (ARSACS) y uno caso de $\mathrm{X}$ frágil. En las ataxias esporádicas lo más frecuente es la AMS-C (13 casos) destacando un gran grupo de pacientes sin diagnóstico.

Las características clínicas de las ataxias neurodegenerativas, se describen en la Figura 2. Es
Tabla 2. Diagnóstico de pacientes con ataxia adquirida

\begin{tabular}{|lcc|}
\hline Diagnóstico & n & Hombre (\%) \\
\hline Psicogénica & 4 & 25 \\
\hline Polineuropatía & 3 & 33,3 \\
\hline Accidente cerebro vascular & 2 & 100 \\
\hline Daño hipóxico isquémico & 1 & 0 \\
\hline Alcoholismo & 2 & 100 \\
\hline Post quirúrgico & 1 & 0 \\
\hline S. cerebeloso autoinmunes & 1 & 0 \\
\hline
\end{tabular}

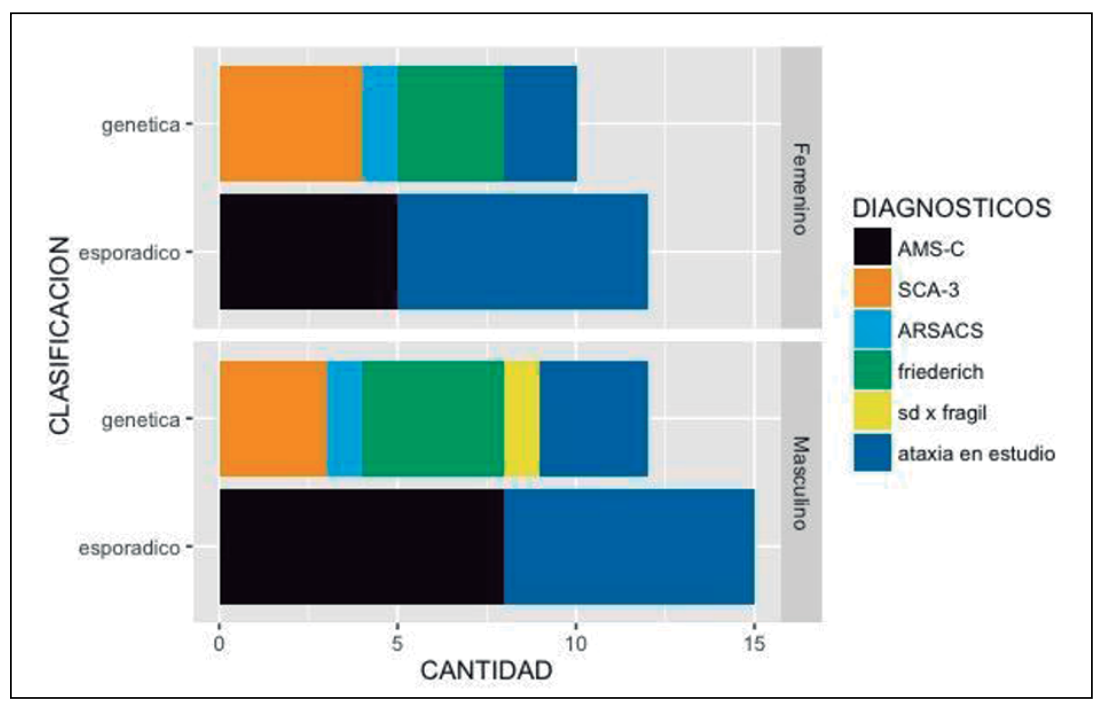

Figura 1. Diagnósticos de pacientes con ataxias esporádicas y genéticas según sexo.

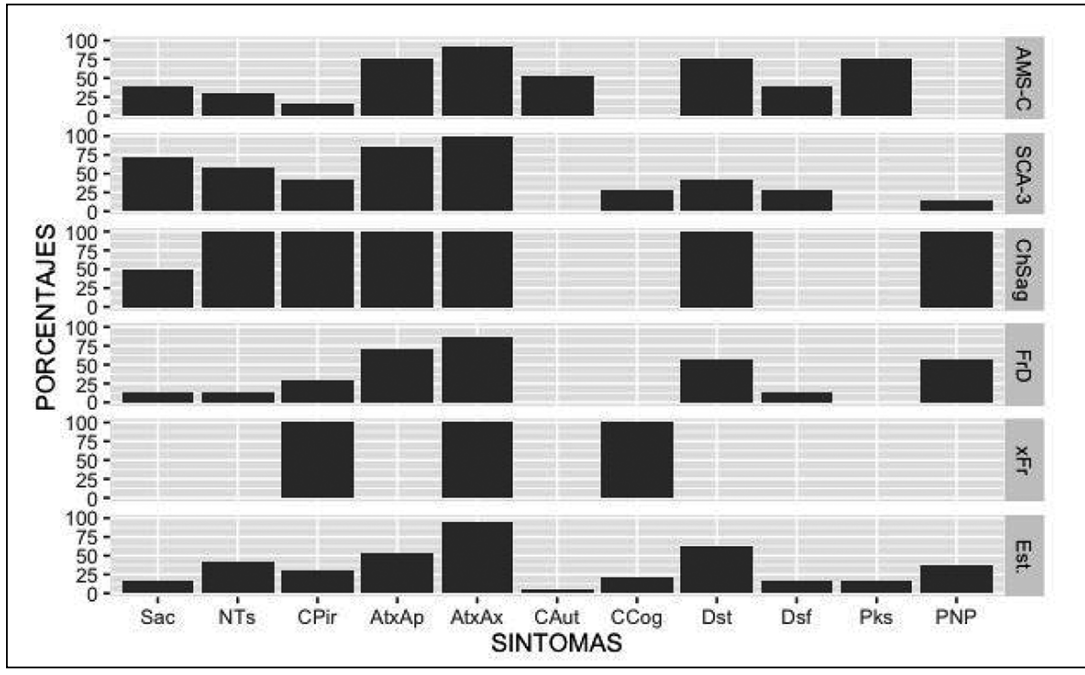

Figura 2. Frecuencia de síntomas según diagnóstico. Sac: alteración en movimientos sacádicos, Nts: Nistagmus, Cpir: compromiso piramidal, AtxAp: ataxia apendicular, AtxAx: ataxia axial, CAut: compromiso autonómico, CCog: compromiso cognitivo, Dst: disartria, Dsf: disfagia, Pks: parkinsonismo, PNP: polineuropatía. ChSag: Charlevoix Saguenay (ARSACS), FrD: Ataxia de Friedreich, $x F r$ : ataxia $X$ frágil, Est.: ataxia en estudio. 
posible observar que ciertos síntomas son de gran ayuda para el diagnóstico como por ejemplo la presencia de parkinsonismo y compromiso autonómico para la AMS-C, o la presencia de polineuropatía que pareciera orientar a una causa genética, sin embargo, hay una gran sobreposición de síntomas.

\section{Discusión}

Esta es la primera serie chilena de ataxias, en un centro especializado en trastornos del movimiento, que compara y analiza sus características clínicas.

A pesar de ser un centro especializado, hubo $31,7 \%$ de los pacientes en los cuales no se logró determinar la causa. Esto es en parte reflejo del hecho de no disponer de estudios para causas genéticas de ataxia en Chile, más aun considerando que en algunos estudios se ha demostrado que hasta $11 \%$ de pacientes clasificados como esporádicos tiene una causa genética ${ }^{6}$. Sin embargo, hasta en los centros con más avanzada tecnología y recursos, siempre queda un porcentaje importante de pacientes sin diagnóstico etiológico ${ }^{6}$.

El comienzo más temprano observado en las ataxias genéticas es algo ya descrito en otras series ${ }^{7}$.

Respecto a los distintos tipos de ataxia genética, encontramos 14 con un patrón autosómico dominante y 9 autosómico recesivo. Entre los autosómico dominantes, con diagnóstico genético, se encontraron sólo casos de SCA 3. Entre los casos con patrón autosómico recesivo, 7 casos de ataxia de Friedreich y 2 casos de ARSACS. Nuestros hallazgos se correlacionan con lo descrito en la literatura, siendo SCA3 de las ataxias autosómicas dominantes más prevalente (aunque varía según regiones) $)^{7,8}$, y la ataxia de Friedreich, la ataxia autosómica recesiva más común?.

$\mathrm{Al}$ considerar el amplio diagnóstico diferencial al enfrentarnos a un cuadro de ataxia, la heterogeneidad de los cuadros clínicos y la gran dificultad de acceder al estudio genético en Chile ${ }^{6}$, proponemos el siguiente esquema de enfrentamiento, que nos ha ayudado en nuestro centro.

La idea es orientar el diagnóstico según el perfil temporal de instalación de la ataxia. De tal forma, para ataxias agudas y subagudas, realizar un estudio agresivo para descartar causas adquiridas como infarto cerebral, encefalopatía de Wernicke, degeneración cerebelosa por alcohol, esclerosis múltiples o fármacos ${ }^{10-12}$.

En los casos subagudos es importante también descartar causas autoinmunes como síndrome paraneoplásico y enfermedades autoinmunes de otra naturaleza, con los exámenes correspondientes según sexo y edad, esto es importante ya que pueden tener implicancias terapéuticas ${ }^{13,14}$.

Los casos de ataxia crónica los podemos dividir según la presencia o ausencia de antecedentes familiares de cuadro similar. Ante la presencia de antecedentes familiares, los más probable es que estemos frente a una ataxia de origen genético. En ausencia de antecedentes familiares es importante buscar compromiso autonómico y parkinsonismo que nos podría orientar al diagnóstico de AMS-C ${ }^{15-17}$. Ante la ausencia de parkinsonismo y compromiso autonómico y aun siendo un cuadro esporádico, hay que pensar en una causa genética. En los casos de ataxia crónica, con antecedentes familiares, es importante intentar determinar el patrón de herencia, que, junto con las características del cuadro clínico, nos pueden orientar a la hora de pedir un estudio genético más específico.

Es importante destacar que las ataxias de etiología autoinmune, también pueden presentar un perfil temporal crónico. En la Tabla 3 sugerimos exámenes útiles para una aproximación diagnostica.

\section{¿Hasta dónde llegar con el diagnóstico?}

Como clínicos, nos vemos frecuentemente enfrentados a este dilema cuando nos toca evaluar pacientes con enfermedades que sabemos, no tienen tratamiento curativo, cómo es el caso de la mayoría de las ataxias neurodegenerativas. Esta es quizá la razón de que no existan en Chile estudios epidemiológicos que den cuenta de este grupo de enfermedades. Las ideas que tenemos de la epidemiología de las ataxias proviene de estudios en otros países, con otras poblaciones, no necesariamente extrapolables a la población chilena. La pregunta no es sólo académica. Sólo a través del conocimiento de cosas tan básicas como la prevalencia de los distintos tipos de ataxia, se podrán crear efectivos programas de apoyo para estos pacientes. Más aún, constantemente sabemos de nuevos genes causantes de ataxia, muchas de estas mutaciones confinadas geográficamente 
Tabla 3. Exámenes sugeridos para el estudio de las ataxias según perfil temporal

\begin{tabular}{|c|c|}
\hline 1. Perfil agudo: & $\begin{array}{l}\text { Resonancia magnética de cerebro } \\
\text { Vitamina B12 y B1 } \\
\text { Hemograma }\end{array}$ \\
\hline 2. Perfil subagudo & $\begin{array}{l}\text { Perfil bioquímico } \\
\text { Fondo de ojo por especialista } \\
\text { Niveles vitamina } \mathrm{E} \\
\text { Cobre y ceruplasmina } \\
\text { Hormonas tiroideas } \\
\text { Anticuerpos } \\
\text { - anti-tiroperoxidasa } \\
\text { - anti-tiroglobulina } \\
\text { - anti-gliadina } \\
\text { - anti-transglutaminasa } \\
\text { Panel paraneoplásico } \\
\text { - Anti-GAD }\end{array}$ \\
\hline 3. Perfil crónico (años) & $\begin{array}{l}\text { EMG, potenciales evocados } \\
\text { Test tolerancia a la glucosa } \\
\text { Ecocardiograma } \\
\text { Electrocardiograma }\end{array}$ \\
\hline $\begin{array}{l}\text { 3.1 Historia familiar compatible con herencia } \\
\text { AD (aparecen entre los } 30 \text { y } 70 \text { años) }\end{array}$ & - Ataxias espinocerebelosas (SCA) 1, 2, 3, 6, 7, 17 \\
\hline $\begin{array}{l}\text { 3.2 Historia familiar compatible con herencia } \\
\text { AR (pueden aparecer a cualquier edad) }\end{array}$ & $\begin{array}{l}\text { - Paso 1: Estudio frataxina, colesteanol, colesterol, alfafetoproteína, albu- } \\
\text { mina } \\
\text { - Paso 2: Estudio mutaciones SACS, Aprataxina, POLG, SPG7 (tomando en } \\
\text { cuenta fenotipo) }\end{array}$ \\
\hline Sospecha específica & $\begin{array}{l}\text { Sospecha de enfermedad de Refsum } \\
\text { Ácido fitanico } \\
\text { Sospecha de adrenoleucodistrofia } \\
\text { Ac Grasos de cadena muy larga } \\
\text { Sospecha de enfermedad mitocondrial } \\
\text { Lactato y piruvato } \\
\text { Sospecha de enfermedad de Sanhoff } \\
\text { Enzimas en glóbulos blancos }\end{array}$ \\
\hline
\end{tabular}

o incluso en pocas familias. El conocimiento de estos nuevos genes es de invaluable utilidad para la comprensión de la fisiopatología de estas enfermedades, y por lo tanto, para eventuales intervenciones terapéuticas.

Podemos aventurar que es muy probable que en la población chilena existan mutaciones no descritas que podrían ser clave para entender mejor las ataxias. Desde el punto de vista de nuestros usuarios, el conocimiento de su genética probablemente permitirá mejorar su manejo, ayudar con consejo genético al paciente y familia, conocer que síntomas pueden surgir en evolución y la sobrevida. Contar con esta información muchas veces contribuye a terminar un largo peregrinar buscando un diagnóstico preciso.
El presente estudio da cuenta de una pequeña muestra de las ataxias evaluadas en un centro especializado en trastornos del movimiento. Urge un estudio multicéntrico que permita caracterizar la epidemiología de las ataxias en Chile.

\section{Referencias}

1. Klockgether T. Sporadic ataxia with adult onset: classification and diagnostic criteria. Lancet Neurol 2010; 9 (1): 94-104.

2. Fahn S, Jankovic J, Hallett M. Principles and practice of movement disorders. Elsevier, 2011.

3. Giordano I, Harmuth F, Jacobi H, Paap B, Vielhaber $\mathrm{S}$, Machts J, et al. Clinical and genetic characteristics 
of sporadic adult-onset degenerative ataxia. Neurology 2017; 89 (10): 1043-9.

4. Muzaimi MB, Thomas J, Palmer-Smith S, Rosser L, Harper PS, Wiles CM, et al. Population based study of late onset cerebellar ataxia in south east Wales. J Neurol Neurosurg Psychiatry 2004; 75 (8): 1129-34.

5. Akbar U, Ashizawa T. Ataxia. Neurol Clin 2015; 33 (1): 225-48.

6. Abele M, Bürk K, Schöls L, Schwartz S, Besenthal I, Dichgans J, et al. The aetiology of sporadic adult-onset ataxia. Brain 2002; 125 (5): 961-8.

7. Jayadev S, Bird TD. Hereditary ataxias: overview. Genet Med 2013; 15 (9): 673-83.

8. Bürk K. Friedreich Ataxia: current status and future prospects. Cerebellum \& Ataxias 2017; 4 (1): 4.

9. Miranda M. Diagnóstico de Ataxia Espinocerebelosa tipo 3 (Enfermedad de Machado-Joseph) en Chile. Rev Med Chile 2015; 143: 126-7.

10. van de Warrenburg BPC, van Gaalen J, Boesch S, Burgunder JM, Dürr A, Giunti P, et al. EFNS/ENS Consensus on the diagnosis and management of chronic ataxias in adulthood. Eur J Neurol 2014; 21 (4): 552-62.

11. van Gaalen J, van de Warrenburg BPC. Republished: A practical approach to late-onset cerebellar ataxia: put- ting the disorder with lack of order into order. Postgrad Med J 2012; 88 (1041): 407-17.

12. Klockgether $\mathrm{T}$. Recent advances in degenerative ataxias. Curr Opin Neurol 2000; 13 (4): 451-5.

13. Mitoma H, Hadjivassiliou M, and Honnorat J. Guidelines for treatment of immune-mediated cerebellar ataxias. Cerebellum \& ataxias 2015; 2 (1): 14.

14. Venkatraman A, Opal P. Paraneoplastic cerebellar degeneration with anti-Yo antibodies - a review. Ann Clin Transl Neurol 2016; 3 (8): 655-63.

15. Wenning GK, Geser F, Krismer F, Seppi K, Duerr S, Boesch $\mathrm{S}$, et al. The natural history of multiple system atrophy: a prospective European cohort study. Lancet Neurol 2013; 12 (3): 264-74.

16. Gatto E, Rodríguez-Violante $\mathrm{M}$, Cosentino C, Chana-Cuevas P, Miranda M, Gallin E, et al. Pan-American Consortium of Multiple System Atrophy (PANMSA). A Pan-American multicentre cohort study of multiple system atrophy. J Parkinsons Dis 2014; 4 (4): 693-8.

17. Gilman S, Wenning GK, Low PA, Brooks DJ, Mathias CJ, Trojanowski JQ, et al. Second consensus statement on the diagnosis of multiple system atrophy. Neurology 2008; 71 (9): 670-6. 